\title{
Upper Limit of Vulnerability During Defibrillator Implantations Predicts the Occurrence of Appropriate Shock Therapy for Ventricular Fibrillation
}

\author{
Soichiro Yamashita, MD; Akihiro Yoshida, MD; Koji Fukuzawa, MD; \\ Ryudo Fujiwara, MD; Atsushi Suzuki, MD; Tomoyuki Nakanishi, MD; \\ Akinori Matsumoto, MD; Hiroki Konishi, MD; \\ Hirotoshi Ichibori, MD; Ken-ichi Hirata, MD
}

\begin{abstract}
Background: The utility of the upper limit of vulnerability (ULV) test in patients undergoing defibrillator implantation has been reported, so the purpose of this study was to evaluate the difference in the clinical outcomes between patients with ULV $\leq 15 \mathrm{~J}$ or $>15 \mathrm{~J}$.

Methods and Results: A total of 165 patients receiving an implantable cardioverter-defibrillator underwent a vulnerability test. At the time of the implantation, we delivered a 15-J shock on the T-peak and $\pm 20 \mathrm{~ms}$ later to cover the most vulnerable part of the cardiac cycle. The clinical outcomes were prospectively analyzed. A 15-J shock induced ventricular fibrillation (VF) in 30 patients (ULV >15 J) and did not in 135 (ULV $\leq 15 \mathrm{~J})$. The characteristics of the 2 groups were comparable. After a mean follow-up of 757 days, Kaplan-Meier curve analysis showed that the ULV $\leq 15 \mathrm{~J}$ group experienced less VF than the ULV $>15 \mathrm{~J}$ group (log-rank $\mathrm{P}=0.003$ ). The occurrence of ventricular tachycardia was similar between the 2 groups $(P=0.140)$. Furthermore, the effectiveness of ATP was comparable. After adjusting for other known predictors of shock therapy, a ULV $>15 \mathrm{~J}$ was independently associated with the occurrence of VF (hazard ratio: 6.25; 95\% confidence interval: 1.913-20.40; $\mathrm{P}<0.01$ ).
\end{abstract}

Conclusions: A high ULV value was associated with a high incidence of VF, which suggests that cardiac vulnerability to electrical shock may be linked to electrical instability. (Circ $J$ 2014; 78: 1606-1611)

Key Words: Defibrillation; Fibrillation; Implantable cardioverter-defibrillator; Shock; Upper limit of vulnerability

$\mathbf{I}$ mplantable cardioverter-defibrillators (ICDs) are the standard therapy for patients at risk of sudden cardiac death (SCD). ${ }^{1-3}$ Defibrillation threshold (DFT) tests have traditionally been part of the ICD implantation to determine a sufficient safety margin. However, the DFT test is associated with the risk of intractable ventricular fibrillation (VF), cerebral hypoperfusion, and myocardial ischemia. The upper limit of vulnerability (ULV) is the weakest shock strength at which VF is not induced when the shock is delivered during the vulnerable period of the cardiac cycle. The correlation between the ULV and DFT has been validated in multiple studies. ${ }^{4-6}$ Clinically, the vulnerability test can be applied to a safetymargin strategy without inducing VF and several protocols have been reported..$^{7,8}$

The mechanism of cardiac vulnerability to electrical shock is still unclear, similar to the mechanism of defibrillation. The purpose of this study was to evaluate the clinical outcome and characteristics of patients with a high ULV in an attempt to better understand the mechanisms of cardiac vulnerability.

\section{Methods}

\section{Study Design}

This was a prospective observational study in order to identify the prespecified effect of the ULV as a predictor of life-threatening arrhythmia occurrence. The primary endpoint was the occurrence of ventricular tachyarrhythmia. We prospectively reviewed 175 consecutive patients who underwent implantation or replacement of an ICD, including a cardiac resynchronization therapy device with defibrillation backup (CRT-D), from February 2009 to December 2012. All patients met the standard guidelines for primary and secondary prevention with an ICD or CRT-D device. ${ }^{9}$ In 165 patients, vulnerability tests were performed at the end of the implantation; 10 patients

Received February 3, 2014; revised manuscript received March 26, 2014; accepted March 27, 2014; released online May 9, 2014 Time for primary review: 20 days

Division of Cardiovascular Medicine, Department of Internal Medicine, Kobe University Graduate School of Medicine, Kobe, Japan

Mailing address: Akihiro Yoshida, MD, Division of Cardiovascular Medicine, Department of Internal Medicine, Kobe University Graduate

School of Medicine, 7-5-2 Kusunoki-cho, Chuou-ku, Kobe 650-0017, Japan. E-mail: ayoshida@med.kobe-u.ac.jp

ISSN-1346-9843 doi:10.1253/circj.CJ-14-0136

All rights are reserved to the Japanese Circulation Society. For permissions, please e-mail: cj@j-circ.or.jp 


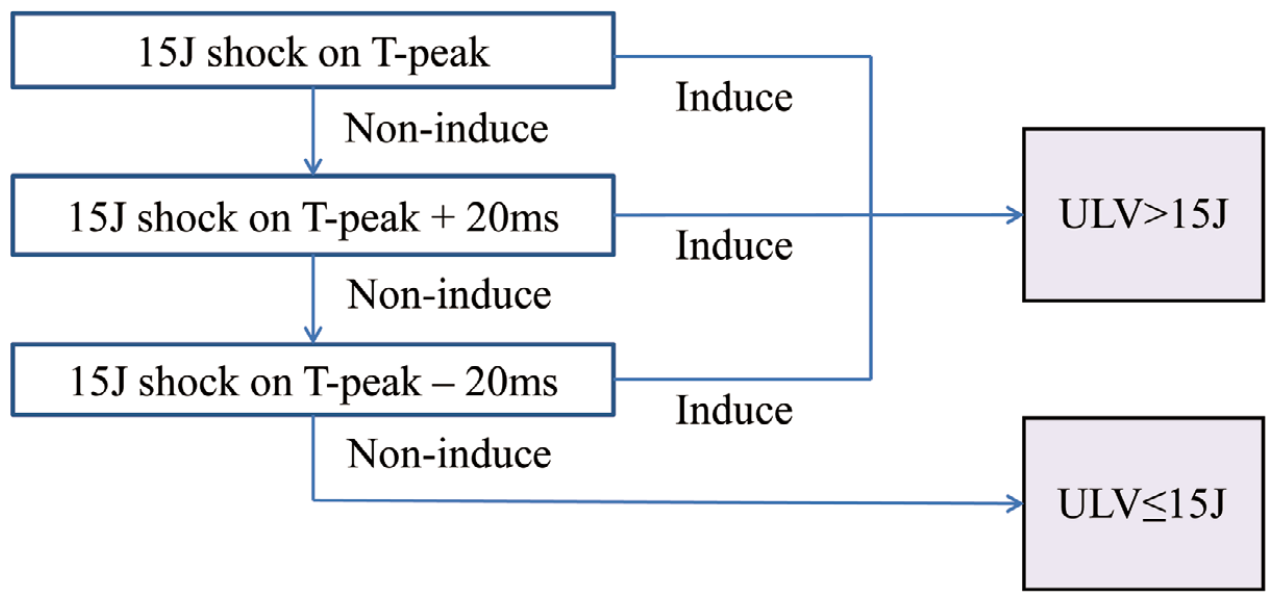

Figure 1. Protocol for our upper limit of vulnerability (ULV) test. The first 15-J T-wave shock was delivered during the St-T peak interval after 8 right ventricular pacing beats with cycle length of $500 \mathrm{~ms}$. If VF was not induced, a subsequent 15-J shock was delivered at $-20 \mathrm{~ms}$ and $+20 \mathrm{~ms}$ from the St-T peak interval. If these procedures did not induce VF, we determined that the ULV was $\leq 15 \mathrm{~J}$. If VF was induced, we determined that the ULV was $>15 \mathrm{~J}$ and defibrillation was tested. VF, ventricular fibrillation.

were excluded because of severe LV dysfunction in 4 , bilateral femoral artery stenosis in 2, alternative DFT testing because of poor right ventricular (RV) sensing in 2, and rejection in 2. Patients who had the device implanted on the right side were also excluded from this study. A baseline clinical history and physical examination were obtained and 12-lead ECG was recorded in each patient. A blood test, including the brain natriuretic peptide (BNP) level, was obtained just before device implantation. Further, echocardiographic studies were performed 10 days before implantation. The left ventricular ejection fraction (LVEF) was assessed by the modified biplane Simpson's method. Medications at the time of the implantation were also noted.

\section{Implantation Technique}

All patients underwent successful implantation or replacement of the ICDs. Commercially available devices (Medtronic, Minneapolis, MN, USA; Boston Scientific, Indianapolis, IN, USA; St. Jude Medical, St. Paul, MN, USA) were used in the trial. The RV leads were all implanted in the RV apex. The choice of a single or dual coil was at the operator's discretion. Written informed consent was given by all patients prior to device implantation.

\section{Vulnerability Test}

We have reported that the 15-J ULV test with 3 coupling intervals could correctly identify high-DFT patients. ${ }^{10}$ All patients were anesthetized with propofol. First, we performed ventricular pacing with 8 pulses at a coupling interval of $500 \mathrm{~ms}$ via the RV lead to measure the stimulus-T wave peak time of the last paced beat to determine the vulnerable period. Second, we delivered a $15-\mathrm{J}$ shock on the T-wave with 3 coupling intervals $(-20,0$, and $+20 \mathrm{~ms}$ from the peak of the Twave). If VF was not induced by these procedures, a ULV $\leq 15 \mathrm{~J}$ was noted and the test was finished. On the other hand, if VF was induced, a ULV $>15 \mathrm{~J}$ was noted, and subsequently we delivered a $25-\mathrm{J}$ shock for defibrillation. If the defibrillation failed, a maximal energy shock was delivered. This protocol is summarized in Figure $\mathbf{1}$ and the details have been described previously. ${ }^{10}$

\section{Device Programming}

The "ventricular tachycardia (VT) zone" and "VF zone" were programmed. For patients in whom VT was not expected to occur, such as those with Brugada syndrome, a single VF zone was programmed. For patients with a history of sustained monomorphic VT, their therapy zones were programmed appropriately to detect each tachycardia. For the VF zone, the devices were programmed to initially deliver the maximal shock therapy. Antitachycardia pacing (ATP) during charging was programmed. Otherwise, for the VT zone, the first therapy was 3-6 ATP sequences. A failed ATP was followed by a shock at $10 \mathrm{~J}$ and then maximal shocks as necessary. In the high-DFT patients, the maximal shock energy was delivered first for each zone. Other specific ATP strategies were determined by the responsible physician. ATP was considered to be unsuccessful when the therapy progressed to an ICD discharge after reaching the maximal number of attempts without termination of the VT.

\section{Follow-up}

The patients were followed in our ICD follow-up dispensary every 3-4 months. Clinical evaluation and device interrogation were carried out at each follow-up visit. The treating physicians were aware of the study-group assignments. Stored intracardiac electrograms (IEGMs) were reviewed by at least 2 independent electrophysiologists using standard criteria to confirm appropriate defibrillator therapy. We evaluate the IEGMs at the time of appropriate therapy and classify the episodes into true VT or true VF based on the IEGMs. True VF was defined as a tachycardia with a cycle length $<300 \mathrm{~ms}$ and the standard deviation (SD) of the V-V interval $>30 \mathrm{~ms}$. If inappropriate ICD therapy occurred and it was necessary to reprogram the device, the physicians could change the device programming.

\section{Statistical Analysis}

Continuous data are presented as the mean \pm SD. Differences 


\begin{tabular}{|c|c|c|c|}
\hline & $\begin{array}{c}U L V \leq 15 J \\
(n=130)\end{array}$ & $\begin{array}{c}U L V>15 J \\
(n=35)\end{array}$ & $P$ value \\
\hline Follow-up, days & $775 \pm 391$ & $717 \pm 402$ & 0.46 \\
\hline Age, years & $64 \pm 14$ & $65 \pm 13$ & 0.84 \\
\hline Male, $n(\%)$ & $98(75)$ & $30(86)$ & 0.19 \\
\hline $\mathrm{BMI}, \mathrm{kg} / \mathrm{m}^{2}$ & $22.6 \pm 3.9$ & $22 \pm 3.1$ & 0.13 \\
\hline $\mathrm{BSA}, \mathrm{m}^{2}$ & $1.63 \pm 0.19$ & $1.65 \pm 0.19$ & 0.69 \\
\hline Primary prevention, $\mathrm{n}(\%)$ & $45(37)$ & $19(54)$ & 0.22 \\
\hline Single coil, $n(\%)$ & $12(9)$ & $5(14)$ & 0.15 \\
\hline NYHA class & $1.8 \pm 0.4$ & $1.7 \pm 0.5$ & 0.52 \\
\hline Ischemic, n (\%) & $48(37)$ & $7(20)$ & 0.06 \\
\hline Nonischemic, n (\%) & $51(39)$ & $18(51)$ & 0.19 \\
\hline HCM, n (\%) & $10(8)$ & $1(3)$ & 0.22 \\
\hline Nonstructural disease, $\mathrm{n}(\%)$ & $17(13)$ & $7(20)$ & 0.3 \\
\hline Other, $\mathrm{n}(\%)$ & $4(3)$ & $2(6)$ & 0.21 \\
\hline CRT-D, n (\%) & $33(25)$ & $14(40)$ & 0.09 \\
\hline $\mathrm{AF}, \mathrm{n}(\%)$ & $29(22)$ & $9(26)$ & 0.67 \\
\hline LVEF, \% & $45 \pm 17$ & $46 \pm 18$ & 0.27 \\
\hline LVDD, mm & $55 \pm 10$ & $56 \pm 12$ & 0.28 \\
\hline LVDS, mm & $43 \pm 14$ & $43 \pm 14$ & 0.29 \\
\hline QRS, ms & $121 \pm 14$ & $119 \pm 12$ & 0.55 \\
\hline Amiodarone, n (\%) & $28(21)$ & $3(8)$ & 0.01 \\
\hline Sotalol, n (\%) & $11(8)$ & $4(11)$ & 0.4 \\
\hline$\beta$-blocker, n (\%) & $106(82)$ & $26(74)$ & 0.34 \\
\hline ACI/ARB, n (\%) & $89(68)$ & $27(77)$ & 0.32 \\
\hline $\mathrm{BNP}, \mathrm{pg} / \mathrm{ml}$ & $196 \pm 77$ & $222 \pm 90$ & 0.22 \\
\hline
\end{tabular}

$\mathrm{ACl}$, angiotensin-converting enzyme inhibitor; $\mathrm{AF}$, atrial fibrillation; $\mathrm{ARB}$, angiotensin II receptor blocker; BMI, body mass index; BNP, brain natriuretic peptide; BSA, body surface area; CRT-D, cardiac resynchronization therapy device with defibrillation backup; HCM, hypertrophic cardiomyopathy; ICD, implantable cardioverter-defibrillator; LVDD, left ventricular end-diastolic dimension; LVDS, left ventricular end-systolic dimension; LVEF, left ventricular ejection fraction; ULV, upper limit of vulnerability.

between mean data were compared using Student's t-test for Gaussian variables. For non-Gaussian variables, the data were compared by the Mann-Whitney test for independent samples, or by Wilcoxon's nonparametric test for paired samples. Categorical data are presented as percentages, and differences in proportions were compared using chi-square analysis. The cumulative event proportions were calculated by Kaplan-Meier analysis, and the differences in the outcome were assessed by a log-rank test. Cox regression analysis was performed to assess the associations between the risk factors at enrolment and the occurrence of VF.

Adjusted hazard ratios of the prespecified predictors of ICD therapy such as age, ${ }^{11}$ NYHA class $,{ }^{12} \mathrm{LVEF},{ }^{13} \mathrm{QRS}$ duration,,${ }^{14}$ BNP concentration, ${ }^{15}$ history of atrial fibrillation (AF),${ }^{16,17}$ indications for secondary prevention, ${ }^{18}$ use of amiodarone and $\beta$-blockers, ${ }^{19}$ and ULV $>15 \mathrm{~J}$ were calculated with Cox proportional hazards regression models. $\mathrm{P}<0.05$ was considered statistically significant. The statistical analyses were performed using SPSS ver. 20.0 software (Chicago, IL, USA).

\section{Results}

\section{Baseline Characteristics}

In this prospective cohort study, a total of 165 patients were enrolled. The median age was 64 years, and $78 \%$ were male. The median NYHA was 1.8 . With regard to the cardiac diseases, $33 \%$ had ischemic and $42 \%$ had nonischemic cardiomyopathy and $15 \%$ had nonstructural heart disease. Other cardiac diseases were valvular heart disease, arrhythmogenic RV cardiomyopathy, vasospastic angina, and congenital heart disease. Overall, $90 \%$ of the patients received a dual-coil ICD and $10 \%$ received a single-coil ICD. The indications for ICD therapy were primary prophylaxis in $39 \%$ and secondary prophylaxis in $61 \%$ of cases. Age, sex, indication for defibrillator therapy, cardiac disease, NYHA class, history of AF, LVEF, QRS width, and BNP level were comparable between the ULV $\leq 15 \mathrm{~J}$ and ULV $>15 \mathrm{~J}$ groups. The only difference between the 2 groups was the use of amiodarone, which was significantly more often prescribed in the ULV $\leq 15 \mathrm{~J}$ group than in the ULV $>15 \mathrm{~J}$ group $(21 \%$ vs. $8 \%, \mathrm{P}<0.05)$ at the time of ICD implantation. A summary of the baseline characteristics is shown in Table 1.

\section{Follow-up}

During $757 \pm 396$ days of follow-up, 12 patients died, but none was SCD. Appropriate shocks and ATPs were documented in 17 and 36 patients, respectively. Of those, 12 true VF episodes were documented. All shocks could terminate the VF on the first attempt, excluding 1 patient in the ULV $\leq 15 \mathrm{~J}$ group who experienced a failure of terminating the VF with maximal shock energy. Fortunately, the VF terminated spontaneously before the third shock. After that event, we re-programmed the tilt and vector of the shock energy, and confirmed the termination of VF by DFT test.

Kaplan-Meier analysis showed that the occurrence of true VF was significantly higher in the ULV $>15 \mathrm{~J}$ group than in 


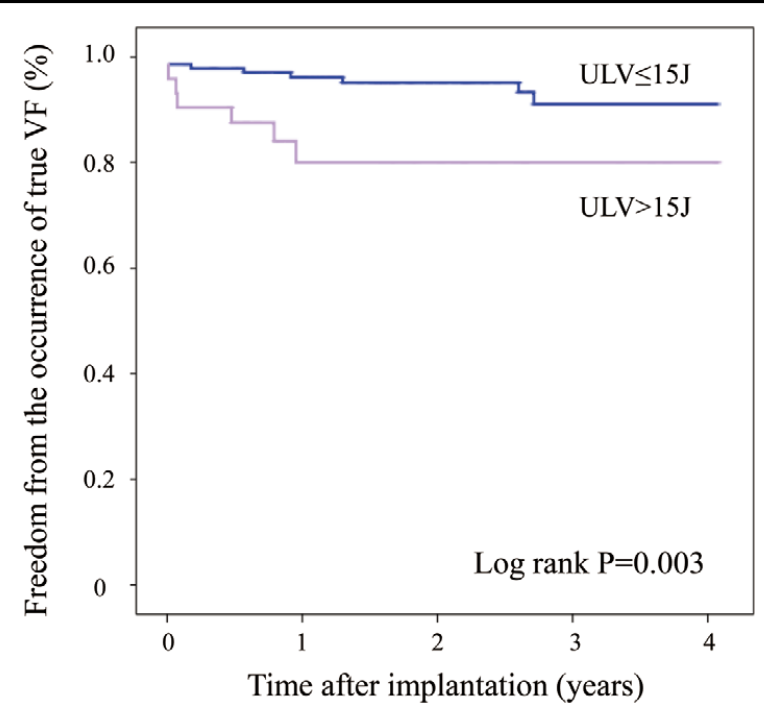

Figure 2. Kaplan-Meier survival estimates for the occurrence of true $V F$ between the $U L V \leq 15 \mathrm{~J}$ group and ULV $>15 \mathrm{~J}$ group. ULV, upper limit of vulnerability; VF, ventricular fibrillation.

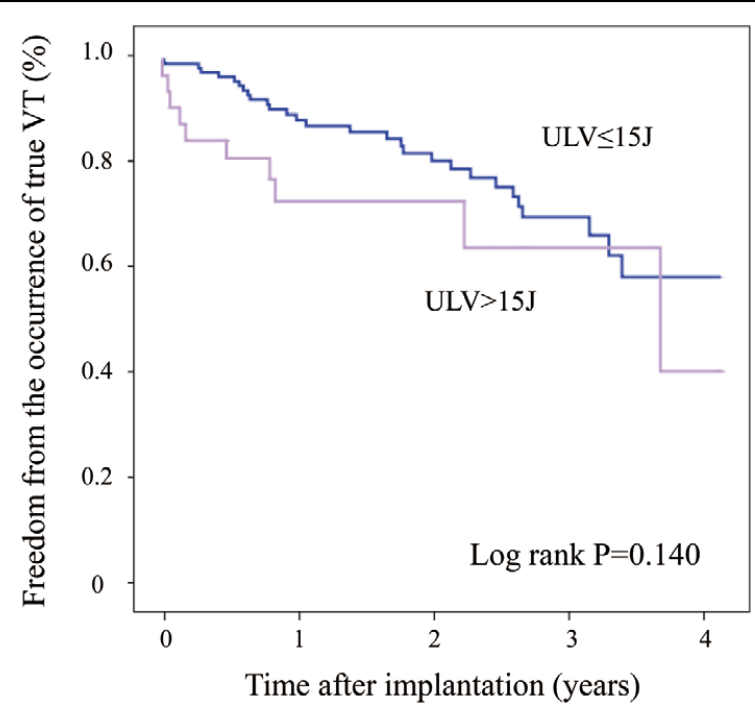

Figure 3. Kaplan-Meier survival estimates for the occurrence of true $V T$ in the ULV $\leq 15 \mathrm{~J}$ and $U L V>15 \mathrm{~J}$ groups. ULV, upper limit of vulnerability; VT, ventricular tachycardia. the $\mathrm{ULV} \leq 15 \mathrm{~J}$ group (Figure 2, log-rank $\mathrm{P}=0.003$ ), despite the occurrence of true VT being comparable between the 2 groups (Figure 3, log-rank $\mathrm{P}=0.140$ ). Multivariate Cox regression analysis revealed that $\mathrm{ULV}>15 \mathrm{~J}$ was an independent predictor of the occurrence of VF (Table 2; hazard ratio: 6.25; $95 \%$ confidence interval: $1.913-20.40 ; \mathrm{P}<0.01)$. The success rate of ATP was comparable between groups (74\% vs. $90 \%$, $\mathrm{P}=0.35$, Table 3 ). VT acceleration by ATP was documented in 2 patients in the ULV $\leq 15 \mathrm{~J}$ group, and in none of the ULV $>15 \mathrm{~J}$ group.

\section{Discussion}

This prospective study demonstrated that ULV $>15 \mathrm{~J}$ was an independent predictor of the occurrence of VF in ICD patients. Further, the occurrence and efficacy of appropriate ATP therapy were similar between the 2 groups.

Our results imply that an increase in the ULV is associated with electrical instability of the ventricle. As far as we know, there has not been a previous clinical report directly showing the relationship between the ULV/DFT and the occurrence of ventricular arrhythmias. The mechanism of cardiac vulnerability to electrical shock is still unclear, although some reports have challenged this difficult problem. Shocks on T-waves produce strong virtual electrode polarizations (VEPs) that re-

\begin{tabular}{|lrrr|}
\hline \multicolumn{4}{|c}{ Table 2. Cox Regression Analysis for the Occurrence of VF } \\
Univariate analysis & EXP & P value & 95\% Cl \\
Age & & & \\
NYHA & 0.95 & 0.01 & $0.924-0.989$ \\
QRS & 0.88 & 0.53 & $0.715-1.885$ \\
Prior documented VF & 0.82 & 0.80 & $0.311-5.022$ \\
ICM & 1.08 & 0.75 & $0.873-11.33$ \\
NICM & 0.92 & 0.56 & $0.512-1.764$ \\
Nonstructural heart disease & 1.08 & 0.66 & $0.438-3.133$ \\
AF & 1.14 & 0.24 & $1.041-2.203$ \\
LVEF & 1.20 & 0.78 & $0.324-4.443$ \\
ULV $>15 \mathrm{~J}$ & 1.01 & 0.54 & $0.976-1.047$ \\
Amiodarone & 4.78 & $<0.01$ & $1.533-14.92$ \\
$\beta$-blocker & 0.37 & 0.31 & $0.122-1.899$ \\
& 0.49 & 0.26 & $0.149-1.659$ \\
Multivariate analysis & HR & $\mathbf{P}$ value & $95 \%$ Cl \\
Age & & & \\
ULV $>15 \mathrm{~J}$ & 0.94 & $<0.01$ & $0.913-0.982$ \\
\hline
\end{tabular}

$\mathrm{Cl}$, confidence interval; ICM, ischemic cardiomyopathy; NICM, nonischemic cardiomyopathy; VF, ventricular fibrillation. Other abbreviations as in Table 1.

\begin{tabular}{|lcccc|}
\hline Table 3. Success Rate of ATP & All & ULV $\leq \mathbf{1 5} \mathbf{J}$ & ULV >15 J & P value \\
Total, $\mathrm{n}$ & 36 & 27 & 9 & \\
Cycle length of true VT, ms & $342 \pm 33$ & $346 \pm 28$ & $339 \pm 45$ & 0.72 \\
1 st sequence success, $\mathrm{n}(\%)$ & $23(63)$ & $16(59)$ & $7(79)$ & 0.31 \\
Termination with more ATP, $\mathrm{n}(\%)$ & $5(14)$ & $4(15)$ & $1(11)$ & 0.78 \\
Spontaneous term, $\mathrm{n}(\%)$ & $6(17)$ & $5(19)$ & $1(11)$ & 0.6 \\
Acceleration, $\mathrm{n}(\%)$ & $2(6)$ & $2(7)$ & 0 & 0.4 \\
Success rate, \% & 77 & 74 & 90 & 0.35 \\
\hline
\end{tabular}

ATP, antitachycardia pacing. Other abbreviations as in Tables 1,2. 
sult in post-shock reentrant arrhythmias via a mechanism of phase singularity. ${ }^{20}$ Anisotropy has been shown to be crucial for the generation of VEPs. ${ }^{21}$ Using a 2-dimensional bi-domain model, Mazeh et al showed that heterogeneity of the fiber angles plays an important role in the mechanisms underlying the ULV. ${ }^{22}$ On the other hand, Maharaj et $\mathrm{al}^{23}$ conducted simulations of shock-induced VF using a 3-dimensional realistic bidomain model and demonstrated that inclusion of transmural heterogeneity in ionic currents results in an increase in the ULV. Propagation proceeds through the post-shock excitable area in the LV free wall, while tissue rendered refractory at the shock-end recovers. Transmural dispersion of the repolarization increases the likelihood of the establishment of reentry within the LV free wall, and explains the increase in the ULV. In another report, the post-shock propagation in the LV free wall was particularly described using a similar model. ${ }^{24}$ Those authors showed that a post-shock activation propagated intramurally through an excitable tunnel in the LV free wall. The fate of the post-shock activation wave might have been related to the propagated intramural graded response and the recovery from the refractory period of the epicardium. Both these reports suggest that anisotropy of the fibers and transmural heterogeneity of the ventricle play key roles in cardiac vulnerability to electrical shock. Both are well-known factors promoting wavebreaks and the generation of fibrillation. ${ }^{25-28}$ The results of our study may arise from these mechanisms. Furthermore, dynamic factors, including the restitution of the action potential duration and conduction velocity, ${ }^{29,30}$ electrotonic currents, ${ }^{31}$ and $\mathrm{Ca}$ cycling properties, ${ }^{32,33}$ have also been recognized as playing an important part in electrical instability. Those factors, together with the tissue heterogeneity, act synergistically to promote wavebreaks and may increase the ULV/DFT.

Multiple protocols of the vulnerability test have been reported. The pacing cycle length, vulnerable period, shock energy, and number of shocks necessarily differ among these reports. ${ }^{7,8,10,34}$ Furthermore, an automated vulnerability safetymargin system has been invented, which measures the T-wave timing using the ECG of the implanted device system. ${ }^{35}$ Similar to those protocols, the high safety and feasibility of our protocol have been reported. ${ }^{10}$ Although differences in the protocol of the vulnerability test might have influenced our results, it may have been relatively small because the peak of the $\mathrm{T}$-wave is in the middle of the vulnerable period in any protocol.

\section{Clinical Implications}

Our results suggest the possibility that VF events may be highly recurrent in patients with an increased ULV. In those patients, an electrical strategy including antiarrhythmic medications, $\beta$-blockers, or catheter ablation should be considered more intensively in clinical practice.

Although a multitude of clinical, laboratory and echocardiographic factors that increase the risk of a high DFT have been identified, it is difficult to predict a high DFT before ICD implantation. Our results point to being able predicting high-DFT patients through the relationship between arrhythmogenicity and vulnerability. Promising noninvasive methods for predicting SCD, such as T-wave alternans, ${ }^{36,37}$ may be related to the ULV/DFT. This possibility merits additional research effort.

\section{Study Limitations}

First, we had a small sample size, so additional studies are required to validate our findings using a larger number of patients in a multicenter trial. Second, the physicians were aware of the study-group assignments during follow-up, so there might have been some bias in the prescribing of drugs that could have influenced the DFT such as amiodarone and $\beta$-blockers. Third, the difference between a single-coil and dual-coil ICD was not considered. In silico, the reaction to a shock on the T-wave differs between a single and dual coil, ${ }^{24}$ which could influence the outcome, although the proportions of single and dual coils was comparable between the 2 groups.

\section{Conclusions}

An increase in the ULV might be an independent predictor of the future occurrence of VF regardless of heart disease. Cardiac vulnerability to electrical shock may be related to electrical instability.

\section{Acknowledgments}

We thank Mitsuaki Itoh, Kimitake Imamura, Kunihiko Kiuchi, and Asumi Takei for their assistance with the data analysis and preparation of the manuscript.

\section{References}

1. The Antiarrhythmics versus Implantable Defibrillators (AVID) Investigators. A comparison of antiarrhythmic-drug therapy with implantable defibrillators in patients resuscitated from near-fatal ventricular arrhythmias. N Engl J Med 1997; 337: 1576-1583.

2. JCS Joint Working Group. Guidelines for non-pharmacotherapy of cardiac arrhythmias (JCS 2011): Digest version. Circ J 2013; 77: 249274.

3. Murakoshi N, Aonuma K. Epidemiology of arrhythmias and sudden cardiac death in Asia. Circ J 2013; 77: 2419-2431.

4. Chen PS, Shibata N, Dixon EG, Martin RO, Ideker RE. Comparison of the defibrillation threshold and the upper limit of ventricular vulnerability. Circulation 1986; 73: $1022-1028$.

5. Hwang C, Swerdlow CD, Kass RM, Gang ES, Mandel WJ, Peter CT, et al. Upper limit of vulnerability reliably predicts the defibrillation threshold in humans. Circulation 1994; 90: 2308-2314.

6. Swerdlow CD, Kass RM, O'Connor ME, Chen PS. Effect of shock waveform on relationship between upper limit of vulnerability and defibrillation threshold. J Cardiovasc Electrophysiol 1998; 9: 339-349.

7. Day JD, Doshi RN, Belott P, Birgersdotter-Green U, Behboodikhah $\mathrm{M}$, Ott P, et al. Inductionless or limited shock testing is possible in most patients with implantable cardioverter-defibrillators/cardiac resynchronization therapy defibrillators: Results of the multicenter ASSURE Study (Arrhythmia Single Shock Defibrillation Threshold Testing Versus Upper Limit of Vulnerability: Risk Reduction Evaluation With Implantable Cardioverter-Defibrillator Implantations). Circulation 2007; 115: 2382-2389.

8. Lemke B, Lawo T, Zarse M, Lubinski A, Kreutzer U, Mueller J, et al. Patient-tailored implantable cardioverter defibrillator testing using the upper limit of vulnerability: The TULIP protocol. Europace 2008; 10: $907-913$.

9. Epstein AE, DiMarco JP, Ellenbogen KA, Estes NA 3rd, Freedman RA, Gettes LS, et al. ACC/AHA/HRS 2008 Guidelines for DeviceBased Therapy of Cardiac Rhythm Abnormalities: A report of the American College of Cardiology/American Heart Association Task Force on Practice Guidelines (Writing Committee to Revise the ACC/ AHA/NASPE 2002 Guideline Update for Implantation of Cardiac Pacemakers and Antiarrhythmia Devices): Developed in collaboration with the American Association for Thoracic Surgery and Society of Thoracic Surgeons. Circulation 2008; 117: e350-e408, doi:10.1161/ CIRCUALTIONAHA.108.189742.

10. Takami K, Yoshida A, Fukuzawa K, Takei A, Kiuchi K, Kanda G, et al. Optimal strength and number of shocks at upper limit of vulnerability testing required to predict high defibrillation threshold without inducing ventricular fibrillation. Circ J 2013; 77: 2490-2496.

11. Kies P, Boersma E, Bax JJ, van der Burg AE, Bootsma M, van Erven $\mathrm{L}$, et al. Determinants of recurrent ventricular arrhythmia or death in 300 consecutive patients with ischemic heart disease who experienced aborted sudden death: Data from the Leiden out-of-hospital cardiac arrest study. J Cardiovasc Electrophysiol 2005; 16: 1049-1056.

12. Whang W, Mittleman MA, Rich DQ, Wang PJ, Ruskin JN, Tofler $\mathrm{GH}$, et al. Heart failure and the risk of shocks in patients with implantable cardioverter defibrillators: Results from the Triggers Of Ventricular Arrhythmias (TOVA) study. Circulation 2004; 109: 13861391. 
13. De Sutter J, Kazmierczak J, Fonteyne W, Tavernier R, Jordaens LJ. Factors determining long-term outcomes and survival in patients with coronary artery disease and ventricular tachyarrhythmias: A single center experience. Pacing Clin Electrophysiol 2000; 23: 1947-1952.

14. Stecker EC, Zargarian M, Dogra V, John BT, Kron J, McAnulty JH, et al. Native QRS duration predicts the occurrence of arrhythmic events in ICD recipients. Europace 2006; 8: 859-862.

15. Verma A, Kilicaslan F, Martin DO, Minor S, Starling R, Marrouche $\mathrm{NF}$, et al. Preimplantation B-type natriuretic peptide concentration is an independent predictor of future appropriate implantable defibrillator therapies. Heart 2006; 92: 190-195.

16. Klein G, Lissel C, Fuchs AC, Gardiwal A, Oswald H, Desousa M, et al. Predictors of VT/VF-occurrence in ICD patients: Results from the PROFIT-Study. Europace 2006; 8: 618-624.

17. Gronefeld GC, Mauss O, Li YG, Klingenheben T, Hohnloser SH Association between atrial fibrillation and appropriate implantable cardioverter defibrillator therapy: Results from a prospective study. J Cardiovasc Electrophysiol 2000; 11: 1208-1214.

18. van Welsenes GH, van Rees JB, Borleffs CJ, Cannegieter SC, Bax JJ, van Erven L, et al. Long-term follow-up of primary and secondary prevention implantable cardioverter defibrillator patients. Europace 2011; 13: 389-394.

19. Connolly SJ, Dorian P, Roberts RS, Gent M, Bailin S, Fain ES, et al. Comparison of beta-blockers, amiodarone plus beta-blockers, or sotalol for prevention of shocks from implantable cardioverter defibrillators: The OPTIC Study: A randomized trial. JAMA 2006; 295: 165 171.

20. Efimov IR, Cheng Y, Van Wagoner DR, Mazgalev T, Tchou PJ. Virtual electrode-induced phase singularity: A basic mechanism of defibrillation failure. Circ Res 1998; 82: 918-925.

21. Efimov IR, Cheng Y, Yamanouchi Y, Tchou PJ. Direct evidence of the role of virtual electrode-induced phase singularity in success and failure of defibrillation. J Cardiovasc Electrophysiol 2000; 11: 861 -868.

22. Mazeh N, Roth BJ. A mechanism for the upper limit of vulnerability. Heart Rhythm 2009; 6: 361-367.

23. Maharaj T, Blake R, Trayanova N, Gavaghan D, Rodriguez B. The role of transmural ventricular heterogeneities in cardiac vulnerability to electric shocks. Prog Biophys Mol Biol 2008; 96: 321 - 338.

24. Ashihara T, Constantino J, Trayanova NA. Tunnel propagation of postshock activations as a hypothesis for fibrillation induction and isoelectric window. Circ Res 2008; 102: 737-745.

25. Delmar M, Michaels DC, Johnson T, Jalife J. Effects of increasing intercellular resistance on transverse and longitudinal propagation in sheep epicardial muscle. Circ Res 1987; 60: 780-785.
26. Akar FG, Rosenbaum DS. Transmural electrophysiological heterogeneities underlying arrhythmogenesis in heart failure. Circ Res 2003; 93: $638-645$.

27. Akar FG, Yan GX, Antzelevitch C, Rosenbaum DS. Unique topographical distribution of $\mathrm{M}$ cells underlies reentrant mechanism of torsade de pointes in the long-QT syndrome. Circulation 2002; 105: $1247-1253$

28. Spach MS, Miller WT 3rd, Geselowitz DB, Barr RC, Kootsey JM, Johnson EA. The discontinuous nature of propagation in normal canine cardiac muscle: Evidence for recurrent discontinuities of intracellular resistance that affect the membrane currents. Circ Res 1981; 48: $39-54$.

29. Qu Z, Weiss JN, Garfinkel A. Cardiac electrical restitution properties and stability of reentrant spiral waves: A simulation study. Am J Physiol 1999; 276: H269-H283.

30. Wu TJ, Lin SF, Weiss JN, Ting CT, Chen PS. Two types of ventricular fibrillation in isolated rabbit hearts: Importance of excitability and action potential duration restitution. Circulation 2002; 106: $1859-1866$.

31. Fenton F, Karma A. Vortex dynamics in three-dimensional continuous myocardium with fiber rotation: Filament instability and fibrillation. Chaos 1998; 8: 20-47.

32. Walker ML, Wan X, Kirsch GE, Rosenbaum DS. Hysteresis effect implicates calcium cycling as a mechanism of repolarization alternans. Circulation 2003; 108: 2704-2709.

33. Diaz ME, O’Neill SC, Eisner DA. Sarcoplasmic reticulum calcium content fluctuation is the key to cardiac alternans. Circ Res 2004; 94: 650-656.

34. Patel MB, Pandya K, Thakur RK. Assessment of adequate safety margin using single coupling interval-upper limit of vulnerability test. Pacing Clin Electrophysiol 2014; 37: 95-103.

35. Birgersdotter-Green U, Ruetz LL, Anand K, Monir G, Abeyratne AI, Bailey JR, et al. Automated vulnerability testing identifies patients with inadequate defibrillation safety margin. Circ Arrhythm Electrophysiol 2012; 5: 1073-1080.

36. Bloomfield DM, Steinman RC, Namerow PB, Parides M, Davidenko J, Kaufman ES, et al. Microvolt T-wave alternans distinguishes between patients likely and patients not likely to benefit from implanted cardiac defibrillator therapy: A solution to the Multicenter Automatic Defibrillator Implantation Trial (MADIT) II conundrum. Circulation 2004; 110: 1885-1889.

37. Hohnloser SH, Ikeda T, Bloomfield DM, Dabbous OH, Cohen RJ. T-wave alternans negative coronary patients with low ejection and benefit from defibrillator implantation. Lancet 2003; 362: 125-126. 